\title{
Evaluation of microRNA-205 expression as a potential triage marker for patients with low-grade squamous intraepithelial lesions
}

\author{
HONG XIE $^{1,2}$, INGRID NORMAN $^{3}$, ANDERS HJERPE $^{4}$, TOMISLAV VLADIC $^{5}$, CATHARINA LARSSON $^{2}$, \\ WENG-ONN LUI ${ }^{2}$, ELLINOR ÖSTENSSON ${ }^{5,6}$ and SONIA ANDERSSON ${ }^{5}$
}

\begin{abstract}
${ }^{1}$ Tianjin Life Science Research Center and Department of Pathogen Biology, School of Basic Medical Sciences, Tianjin Medical University, Tianjin 300070, P.R. China; ${ }^{2}$ Department of Oncology-Pathology, Karolinska Institutet, Cancer Center Karolinska, Karolinska University Hospital Solna, 17176 Stockholm; ${ }^{3}$ Department of Clinical Science,

Intervention and Technology, Division of Obstetrics and Gynecology, Karolinska University Hospital Huddinge;

${ }^{4}$ Department of Laboratory Medicine, Division of Pathology, Karolinska Institutet, Karolinska University Hospital Huddinge,

14186 Stockholm; ${ }^{5}$ Department of Women's and Children's Health, Division of Obstetrics and Gynecology,

Karolinska Institutet, Karolinska University Hospital Solna, 17176 Stockholm;

${ }^{6}$ Department of Medical Epidemiology and Biostatistics, Karolinska Institutet, 17177 Stockholm, Sweden
\end{abstract}

Received October 3, 2016; Accepted January 26, 2017

DOI: 10.3892/ol.2017.5909

\begin{abstract}
High-risk human papillomavirus (HPV) testing is a recommended triage approach for females with atypical squamous cells of undetermined significance (ASCUS), but due to its poor specificity this approach is not recommended for patients with low-grade squamous intraepithelial lesions (LSIL). The objective of the current study was to determine microRNA (miR)-205 expression levels in liquid-based cytology (LBC) samples, and evaluate their ability to predict cervical intraepithelial neoplasia grade $2 / 3$ or worse (CIN2/3+) in females with minor cytological abnormalities. LBC samples
\end{abstract}

Correspondence to: Dr Sonia Andersson, Department of Women's and Children's Health, Division of Obstetrics and Gynecology, Karolinska Institutet, Elevhemmet H2:00, Karolinska University Hospital Solna, 17176 Stockholm, Sweden

E-mail: sonia.andersson@karolinska.se

${ }^{*}$ Contributed equally

Abbreviations: ASCUS, atypical squamous cells of undetermined significance; CI, confidence interval; CIN, cervical intraepithelial neoplasia; CIN1, cervical intraepithelial neoplasia grade 1; CIN2, cervical intraepithelial neoplasia grade 2; CIN2+, cervical intraepithelial neoplasia grade 2 or worse; CIN3+, cervical intraepithelial neoplasia grade 3 or worse; HPV, human papillomavirus; HSIL, high-grade squamous intraepithelial lesion; LBC, liquid-based cytology; LSIL, low-grade squamous intraepithelial lesions; miRNA, microRNA; RT-qPCR, reverse transcription-quantitative polymerase chain reaction; WNL, within normal limits (normal cytology)

Key words: liquid-based cytology, microRNA-205, specificity, human papillomavirus, cervical intraepithelial lesions were obtained from patients attending the Swedish Cervical Cancer Screening Program. The Mann-Whitney U test, one-way analysis of variance, Kruskal-Wallis test, Spearman rank order correlation analysis, and Pearson's $\chi^{2}$ test were used to assess the results. Accuracy analyses indicated that high miR-205 expression had a significantly higher specificity to high-risk HPV testing, and a sensitivity similar to that of high-risk HPV testing to predict CIN2+ and CIN3+ in women with LSIL, but not those with high-grade squamous intraepithelial lesions. Although further research is required for females with LSIL, miR-205 expression in LBC samples may be a novel triage marker for, or a beneficial supplement to high-risk-HPV testing in these patients.

\section{Introduction}

Cervical cancer is a leading cause of cancer-associated mortality among females worldwide. It accounts for $13 \%$ of all female cancer cases, with $>500,000$ new cases and 275,000 mortalities occurring annually (1). In Sweden, 450 new cases and 150 mortalities occur each year (2). According to reports from the organized Swedish Cervical Cancer Screening Program, 30,000 women exhibit some form of cellular abnormality and require follow-up with colposcopy and biopsy (3).

Persistent infection with human papillomavirus (HPV) is the causative agent in cervical cancer (4). HPV depends on differentiated keratinocytes; the infection of the squamous epithelia alone is not sufficient for the infection to progress to neoplasia (5). The expression of the HPV oncoproteins E6 and E7 is able to inactivate p53 and retinoblastoma proteins, leading to methylation and mutation of the host genome DNA and resulting in the initiation of and progression towards cancer $(6,7)$. The use of high-risk HPV (8) testing in primary screening for cervical disease has exhibited a high 
sensitivity (9), but the specificity of this method is low, and thus a follow-up test must be administered prior to treatment (10).

The implementation of organized cervical cancer screening programs has reduced the incidence of cervical cancer considerably (11). However, several previous studies have demonstrated that conventional cytology has a limited sensitivity (only 50-70\%) to detect cervical intraepithelial neoplasia (CIN) $(12,13)$. Liquid-based cytology (LBC) was developed to improve diagnostic reliability (14), as it offers the possibility to use the same sample for HPV testing and triage. Such triage is recommended for women with atypical squamous cells of undetermined significance (ASCUS) due to its high sensitivity, but it is not recommended for women with low-grade squamous intraepithelial lesions (LSIL) due to the high prevalence of high-risk HPV in this population, which generally leads to poor specificity (15). The low predictive value of HPV testing among females with minor cytological abnormalities may create unnecessary concern among healthy patients and contribute to a significant risk of over-diagnosis and over-treatment. The use of predictive biomarkers is a novel approach to improving the diagnosis and management of patients with LSIL.

MicroRNA (miRNA) is a small, non-coding RNA that is $\sim 22$ nucleotides in length. miRNA has an important role in pathological processes, including viral infection and cancer development (4). Generally, miRNA negatively regulates gene expression at the post-transcriptional level via transcription inhibition and/or translation suppression (16). Previous studies have identified altered miRNA expression profiles in human cervical cancer tissues and cell lines, and several of them, including miRNA (miR)-145, miR-21 and miR-205, are consistently dysregulated in cervical cancer tissue compared with normal cervical tissue (17-19). In our previous study, it was revealed that miR-205 expression was significantly increased in cervical cancer tissue compared with matched normal cervical tissue, and that miR-205 has an oncogenic role in cervical cancer through the promotion of cell proliferation and migration (20). This prompted the further investigation of the potential value and clinical applications of miR-205 in the present study.

Recently, miRNAs were suggested as potential biomarkers for the diagnosis or prognosis of different cancer types, including cervical cancer (21-24). Due to the requirement for non-invasive detection methods, the majority of the applications focused on serum or plasma samples. For example, serum miR-203 expression was an independent predictive marker for lymph node, peritoneal and distant metastases, and a poor prognosis marker in patients with gastric cancer (8). In patients with colorectal cancer, circulating miR-103, miR-720 and miR-372 were potential novel biomarkers: High serum miR-103 expression levels were significantly associated with histological differentiation grade and lymphatic invasion; high serum miR-720 levels were significantly associated with lymph node metastasis; and high miR-372 levels were significantly associated with tumor size, tumor-node-metastasis stage and poorer overall survival $(25,26)$. Downregulation of miR-205 expression in colorectal cancer predicts the risk of lymph node metastasis (27). Circulating miR-205 and let-7f together were reported to be diagnostic biomarkers for ovarian cancer (28). Serum miR-205 expression was revealed to be significantly downregulated in patients with glioma compared with healthy controls and was a novel and valuable biomarker for the diagnosis of glioma, and a prognostic factor for those with advanced-grade tumors (29). Ma et al (30) reported that upregulated serum miR-205 is a predictive marker for the prognosis of cervical cancer, and Zhao et al (31) reported that high circulating miR-20a expression levels represent a potential marker for detecting lymph node metastasis in early-stage cervical cancer. However, only a limited number of studies have performed miRNA detection in cervical exfoliated cells $(32,33)$.

The aim of the present study was to investigate whether miR-205 expression may be used as a novel triage approach to predict high-grade CIN in LBC samples from patients attending the population-based Swedish Cervical Cancer Screening Program.

\section{Materials and methods}

Study population. Between 2008 and 2012, LBC samples were collected from 140 women with squamous intraepithelial lesions or squamous cell carcinoma detected within the framework of the Swedish Cervical Cancer Screening Program in Stockholm, Sweden (34). Cervical cells for LBC were obtained from the ectocervix and endocervix of the uterus, preserved in PreservCyt medium (ThinPrep ${ }^{\circledR}$, Hologic, Boxborough, MA, USA) at $-20^{\circ} \mathrm{C}$, and evaluated at the Department of Clinical Pathology and Cytology, Karolinska University Hospital (Solna-Stockholm, Sweden). Cytological results were categorized according to the Bethesda classification (35), with modifications based on Swedish recommendations: Samples with coilocytosis, but without cellular atypia, were classified as 'within normal limits' (WNL), and LSIL included mild dysplasia only. The diagnosis and staging of CIN was based on colposcopy and histology, and grouped into normal histology (WNL), CIN grade 1 (CIN1), CIN grade 2 (CIN2) and CIN2 or worse (CIN2+). Histological information and high-risk-HPV test results were retrieved from the medical and laboratory records at the Karolinska University Hospital.

This study was approved by the Ethical Review Board at Karolinska Institutet (Stockholm, Sweden) and written informed consent was obtained from all participants prior to sample collection.

RNA extraction. Cervical cells were collected by centrifugation and washed with cold PBS twice, followed by total RNA extraction using the mirVana ${ }^{\mathrm{TM}}$ miRNA isolation kit (Thermo Fisher Scientific, Inc., Waltham, MA, USA), all according to the manufacturer's protocol. RNA concentrations were measured using a NanoDrop ND-1000 spectrophotometer (NanoDrop Technologies, Wilmington, DE, USA) and stored at $-80^{\circ} \mathrm{C}$ for further use.

TaqMan RT-qPCR. miR-205 expression was quantified by TaqMan reverse transcription quantitative polymerase chain reaction (RT-qPCR) using the StepOne Plus real-time PCR system (Thermo Fisher Scientific, Inc.). cDNA was synthesized from $100 \mathrm{ng}$ of RNA using the TaqMan miRNA reverse transcription kit (Applied Biosystems; Thermo Fisher Scientific, Inc.). The pre-designed TaqMan assays for miR-205 
(ID 000509) and the reference material RNU6B (ID 001093) were purchased from Thermo Fisher Scientific, Inc. (20). All reactions were performed in triplicate, according to the manufacturer's protocol. The relative expression of miR-205 was normalized to RNU6B and reported as $2^{-\Delta \Delta \mathrm{Cq}}(36)$.

HPV DNA detection. HPV testing was performed at Karolinska University Hospital. Briefly, DNA was extracted from the LBC suspensions using the MagNA Pure LC Robot (Roche Diagnostics, Basel, Switzerland). HPV DNA detection and genotyping were carried out using the Linear Array HPV Genotyping test (Roche Diagnostics, Mannheim, Germany) and Cobas 4800 (Roche Diagnostics, Basel, Switzerland), which detects 37 HPV types: High-risk-HPV types (HPV16, $18,31,33,35,39,45,51,52,56,58,59 / 68 / 73$, and 82 ); probable high-risk-HPV types (HPV26, 53, and 66); and low-risk or undetermined-risk HPV types (HPV6, 11, 40, 42, 43, 44, 54, $55,61,62,64,67,69,70,71,72,81,83,84$, IS39, and CP6108).

Statistical analysis. Data were entered into Statistica 7.0 (Statsoft, Inc., Tulsa, OK, USA). The difference in miR-205 expression between all HPV-positive and all HPV-negative samples was analyzed using the Mann-Whitney U test. The associations between miR-205 expression levels and diagnoses (including cytology, histology and the final histopathological diagnosis) were analyzed by the Kruskal-Wallis one-way analysis of variance (ANOVA) test. The correlation of miR-205 expression with age was analyzed with the Spearman Rank Order correlation and Pearson's $\chi^{2}$ test. Sensitivity and specificity calculations were performed using VassarStats online software (http://vassarstats.net/). $\mathrm{P}<0.05$ was considered to indicate a statistically significant difference.

\section{Results}

Cytology, histology, final diagnosis and HPV status. The median age of the 140 females in the study sample was 32.5 years (range, 23-59 years). Of these patients, 123 (123/140, $87.9 \%)$ had histological information available, and $115(115 / 140$, 82.1\%) had HPV test results available in the medical and laboratory records at the Karolinska University Hospital. Among the patients with HPV results, 93 were HPV-positive (93/115, $80.9 \%$ ) and 22 were HPV-negative $(22 / 115,19.1 \%)$ (Table I).

Of the 93 HPV-positive women, only one (no. 43) was infected with a low-risk HPV type (HPV54). Eighty-seven patients were infected with at least one high-risk HPV type, and $43(43 / 93,46.2 \%)$ were infected with either HPV16 or 18, the two most common high-risk HPV types (Table II).

Sensitivity and specificity of high miR-205 expression levels to predict CIN2+ and CIN3+ in LSIL and HSIL. Sensitivity and specificity analyses were performed among patients with LSIL and high-grade squamous intraepithelial lesions (HSIL), based on high miR-205 expression levels and HPV positivity. The specificity of HPV testing to predict the absence of CIN2+ and cervical intraepithelial neoplasia grade 3 or worse (CIN3+) was 0.11 [95\% confidence interval (CI), 0.03-0.30] and 0.08 (95\% CI, 0.02-0.23), respectively, in women with LSIL. The specificity of high miR-205 expression levels was 0.63 (95\% CI, 0.42-0.80) and 0.57 (95\% CI, 0.40-0.72), which
Table I. Summary of clinical features of the study sample $(\mathrm{N}=140)$.

\begin{tabular}{lrr}
$\begin{array}{l}\text { Characteristic } \\
\text { (N with results available) }\end{array}$ & N \\
\hline Cytology (N=140) & & \\
WNL & 18 & 12.86 \\
LSIL & 45 & 32.14 \\
HSIL & 74 & 52.86 \\
Cancer & 3 & 2.40 \\
Histology (N=123) & & \\
WNL & 9 & 7.32 \\
CIN1 & 35 & 28.46 \\
CIN2 & 28 & 22.76 \\
CIN3 & 47 & 38.21 \\
Cancer & 4 & 3.25 \\
Final histopathological & & \\
diagnosis (N=140) & & \\
WNL & 16 & 11.43 \\
CIN1 & 29 & 20.71 \\
CIN2 & 44 & 31.43 \\
CIN3 & 47 & 33.57 \\
Cancer & 4 & 2.86 \\
HPV testing (N=115) & & \\
Positive & 93 & 80.87 \\
Negative & 22 & 19.13 \\
\hline
\end{tabular}

$\mathrm{N}$, number; WNL, within normal limits (normal cytology); LSIL, low-grade squamous intraepithelial lesion; HSIL, high-grade squamous intraepithelial lesion; CIN1, cervical intra-epithelial neoplasia grade 1; CIN2, cervical intra-epithelial neoplasia grade 2; CIN3, cervical intra-epithelial neoplasia grade 3; HPV, human papillomavirus.

was significantly higher than that of HPV testing. Although positivity for HPV16, HPV18, or HPV16/18 exhibited a higher sensitivity $(0.88,0.96$, and 0.85 , respectively, to predict CIN2+; $0.83,0.94$, and 0.73 , respectively, to predict CIN3+) than high miR-205 expression levels, these values were not statistically significant (Table III).

Although the specificity of HPV testing to predict CIN3+ in patients with HSIL was lower than that of high miR-205 expression levels $(0.16,95 \%$ CI: $0.05-0.37 ; 0.38,95 \%$ CI, $0.23-0.56$, respectively), this trend was also not statistically significant (Table IV).

The sensitivity of high miR-205 expression to predict CIN2+ and CIN3+ was 0.56 (95\% CI, 0.31-0.78) and 0.50 (95\% CI, 0.17-0.83), respectively, among patients with LSIL, whereas HPV testing had a corresponding sensitivity of 1.0 (95\% CI, 0.78-1) and 1.0 (95\% CI, 0.60-1), respectively. Furthermore, when divided by HPV type, the individual sensitivity values $(0.33,0.11$ and 0.44 for CIN2+; $0.38,0.12$ and 0.50 for CIN3+) were not higher than those for high miR-205 expression levels; the ANOVA test revealed that the differences between HPV testing and high miR-205 expression levels 


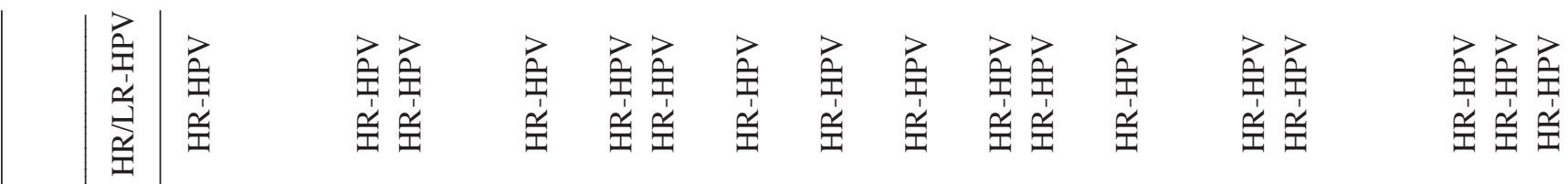

完

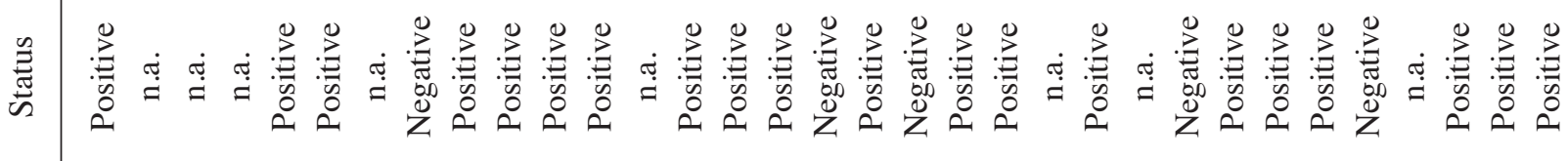

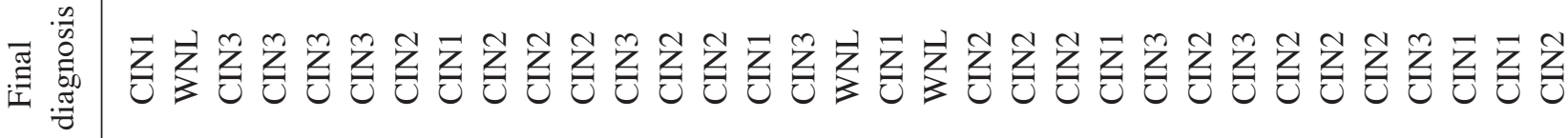

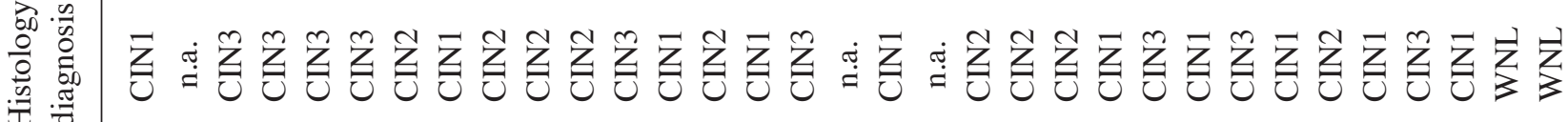

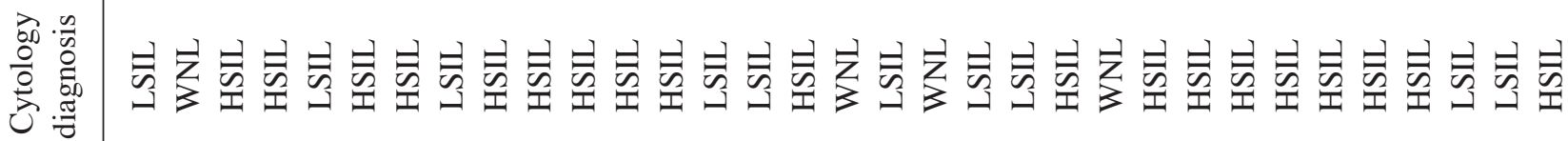

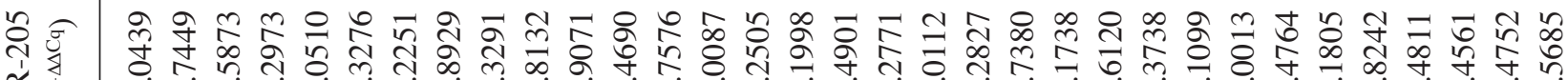
光

ষ্ৰ 


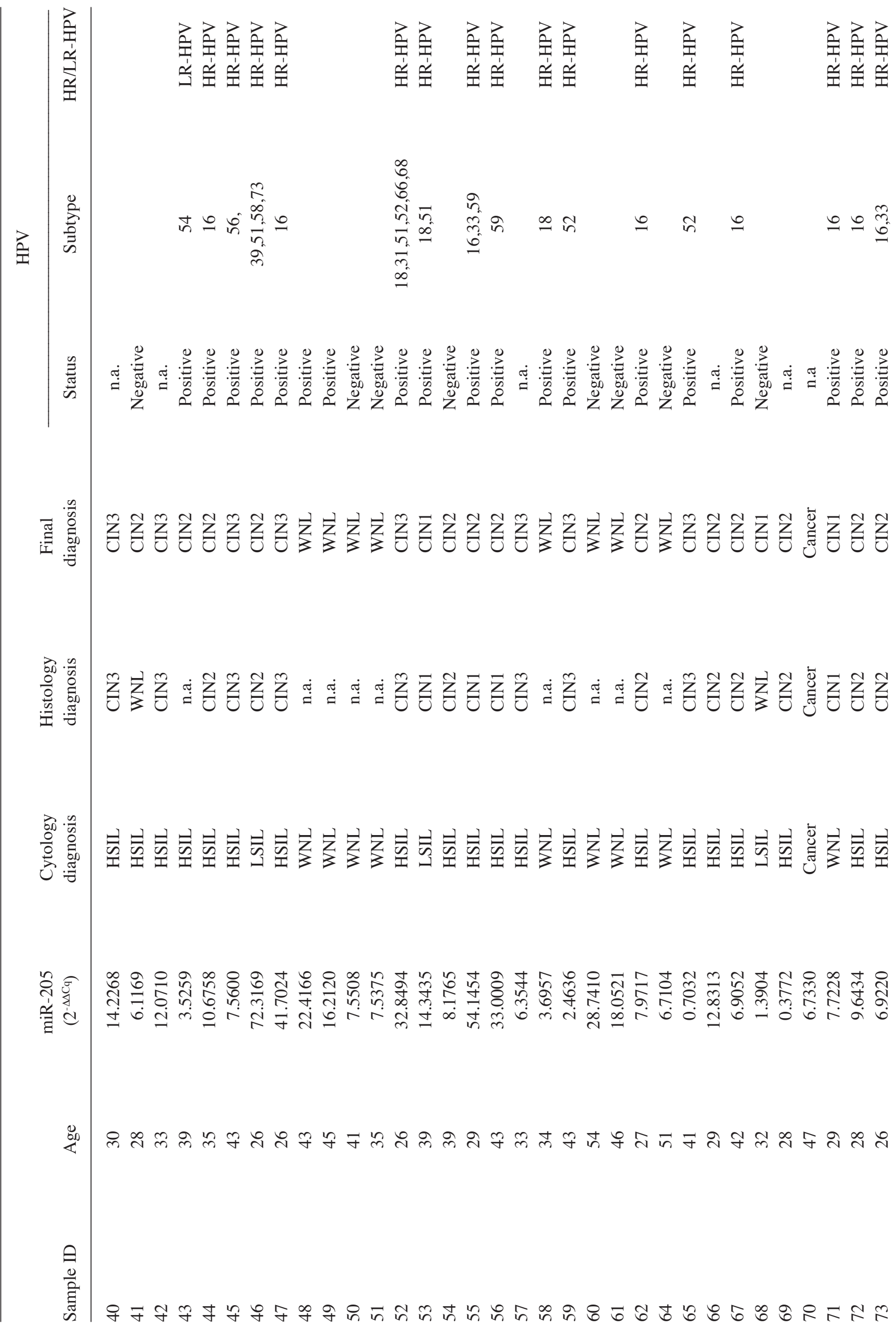




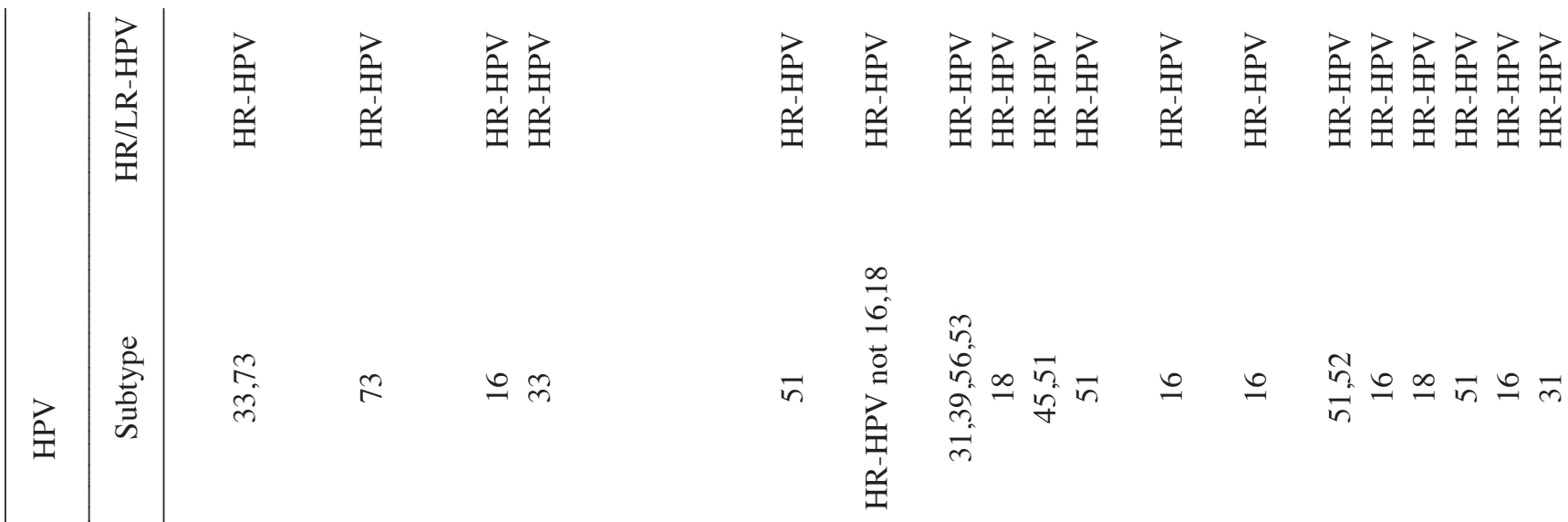

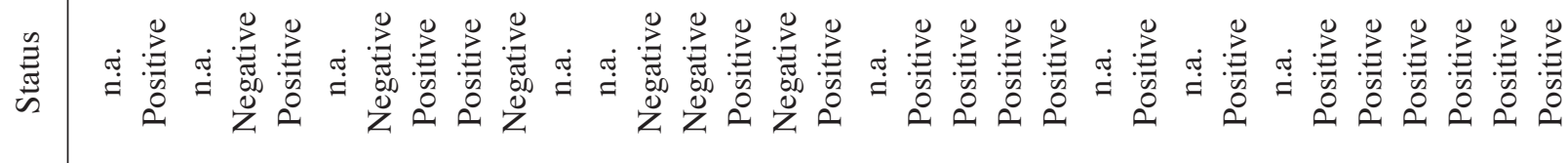

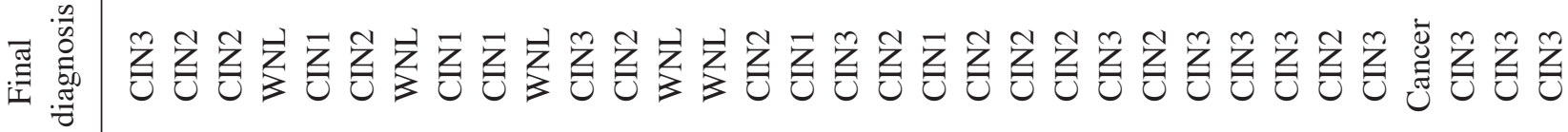

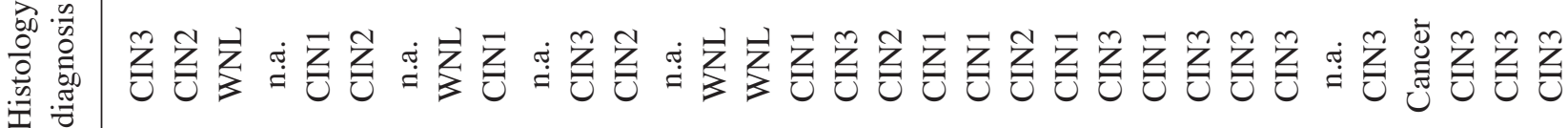

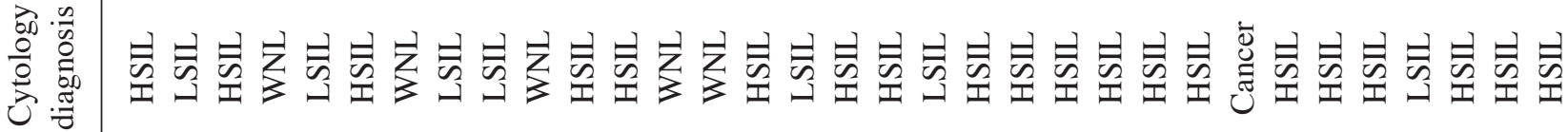

商 当

㐫 


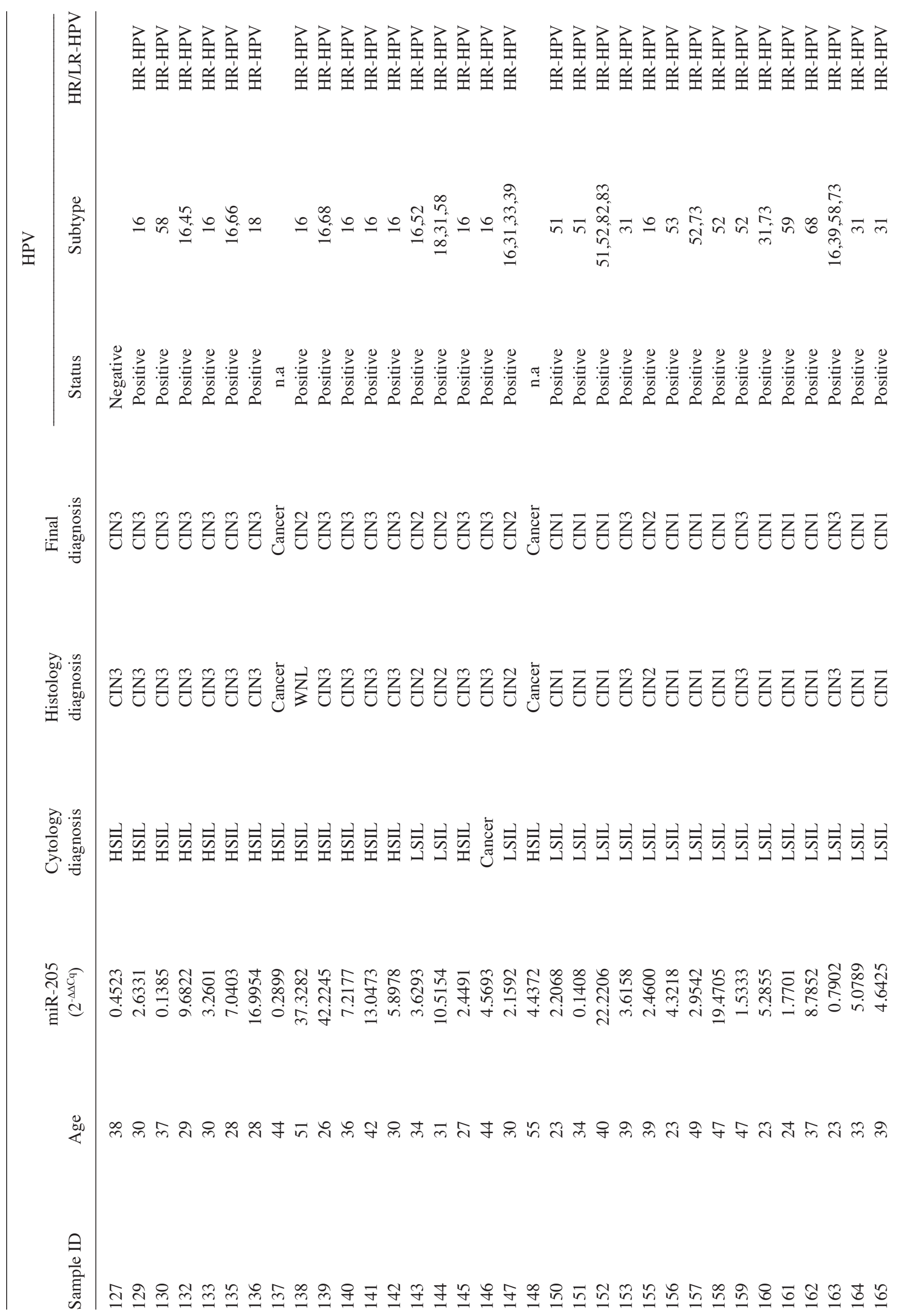


were not statistically significant (Table III). Similar results were obtained in the HSIL group, in which the sensitivity of HPV testing to predict CIN2+ and CIN3+ was 0.87 (95\% CI, 0.74-0.94) and 0.89 (95\% CI, 0.71-0.97), respectively, which was higher than that of high miR-205 expression levels $(0.55$, 95\% CI, 0.43-0.67 for CIN2+ and 0.50, 95\% CI, 0.34-0.66 for CIN3+; Table IV).

miR-205 expression is not associated with HPV status, but may differ by HPV type. Using the relative quantification method $\left(2^{-\Delta \Delta \mathrm{Cq}}\right)$, as normalized to RNU6B, the relative miR-205 expression in all 140 LBC samples was calculated, and the associations between miR-205 expression and HPV positivity in the 115 samples that had this information available were analyzed using the Mann-Whitney $U$ test. No statistically significant difference in miR-205 expression was observed between HPV-positive $(n=93)$ and HPV-negative $(n=22)$ samples $(\mathrm{P}=0.97$; $\mathrm{Z}$-score $=0.039$; two-tailed $)$, indicating that miR-205 expression was not associated with HPV positivity. Similar results were obtained using the $\chi^{2}$ test (Table V). A univariate test for miR-205 expression in all 140 samples revealed significant differences $\left(\mathrm{P}=1 \times 10^{-6}\right)$, indicating the role of an unknown variable. Therefore, the association between miR-205 expression and HPV type, particularly HPV16 and 18, was investigated using the ANOVA Kruskal-Wallis test. Although the mean miR-205 expression levels in HPV18-positive samples (mean value, 18.98; $n=9$ ) were higher than those in HPV16-positive samples (mean value, 12.27; $\mathrm{n}=34$ ), due to small sample size and large variation between samples, they were not statistically significant $(\mathrm{P}=0.279)$.

miR-205 expression and age. Spearman Rank Order correlation analyses did not reveal any significant correlations between miR-205 expression and age $(\mathrm{R}=-0.0836 ; \mathrm{P}=0.324)$; similar results were obtained using $\chi^{2}$ tests (Table $\mathrm{V}$ ).

miR-205 expression and cervical cancer progression. No significant difference between the LSIL and the HSIL group was observed based on cytology diagnosis, histology diagnosis or final histopathological diagnosis $(\mathrm{P}=0.64,0.70$ and 0.32 , respectively), indicating that miR-205 expression alone was not able to distinguish the progression of cervical cancer in LBC samples. Based on the median expression levels of miR-205 in the 140 LBC samples, the correlations between $m i R-205$ expression and different characteristics, including age, HPV positivity, HPV type, and final histopathological diagnosis were evaluated using a two-tailed $\chi^{2}$ test; however, no significant differences were observed (Table V).

\section{Discussion}

Cervical cancer develops from well-recognized, pre-malignant forms. The detection of these forms through population-based screening programs is able to reduce the number of cases of cervical cancer dramatically (37). However, more robust and reliable molecular markers are required in current screening programs in order to distinguish between lesions with invasive potential and lesions that will spontaneously regress.

miRNAs are well described non-coding RNAs involved in human cancer, which typically negatively regulate gene 


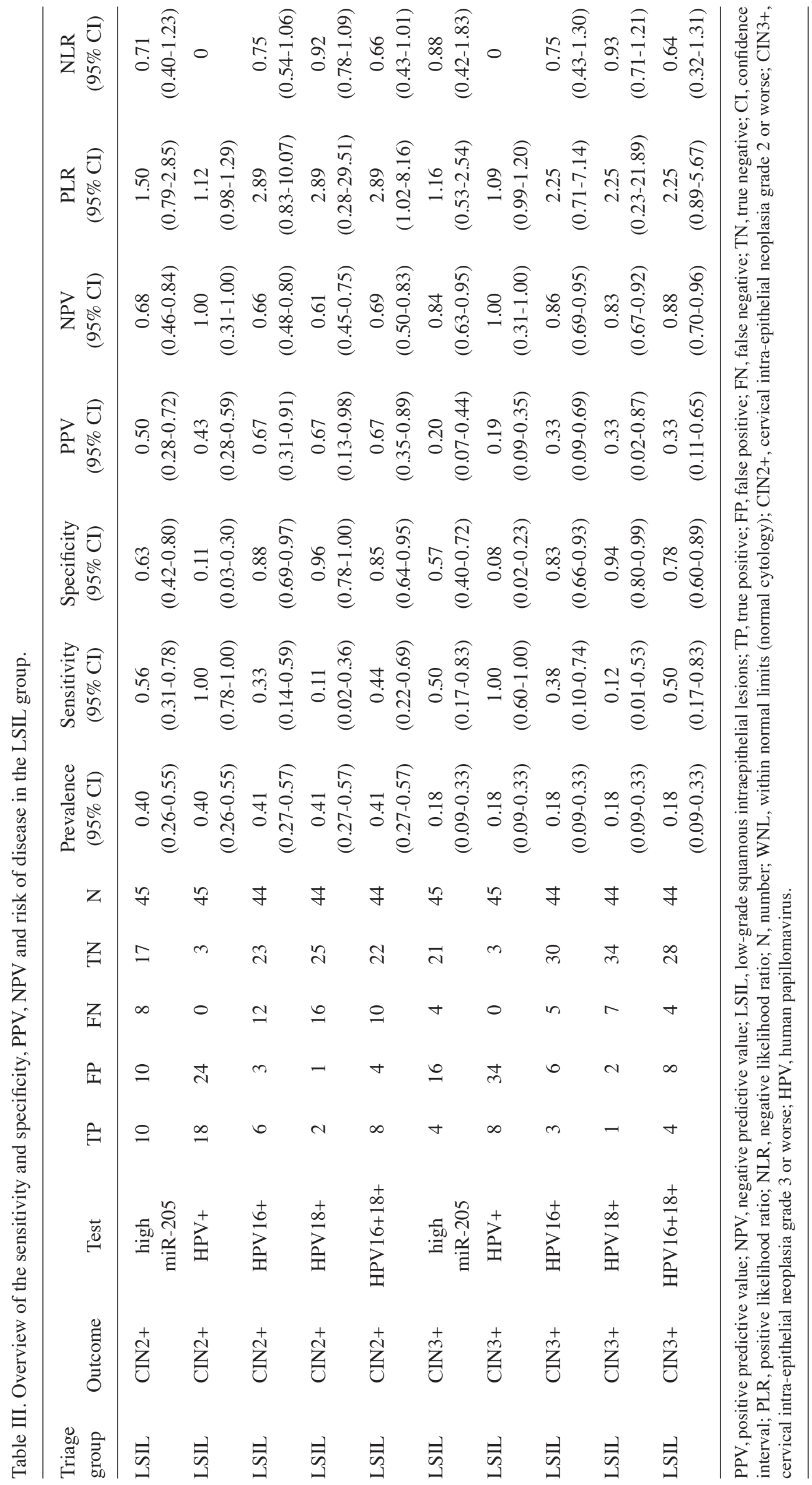




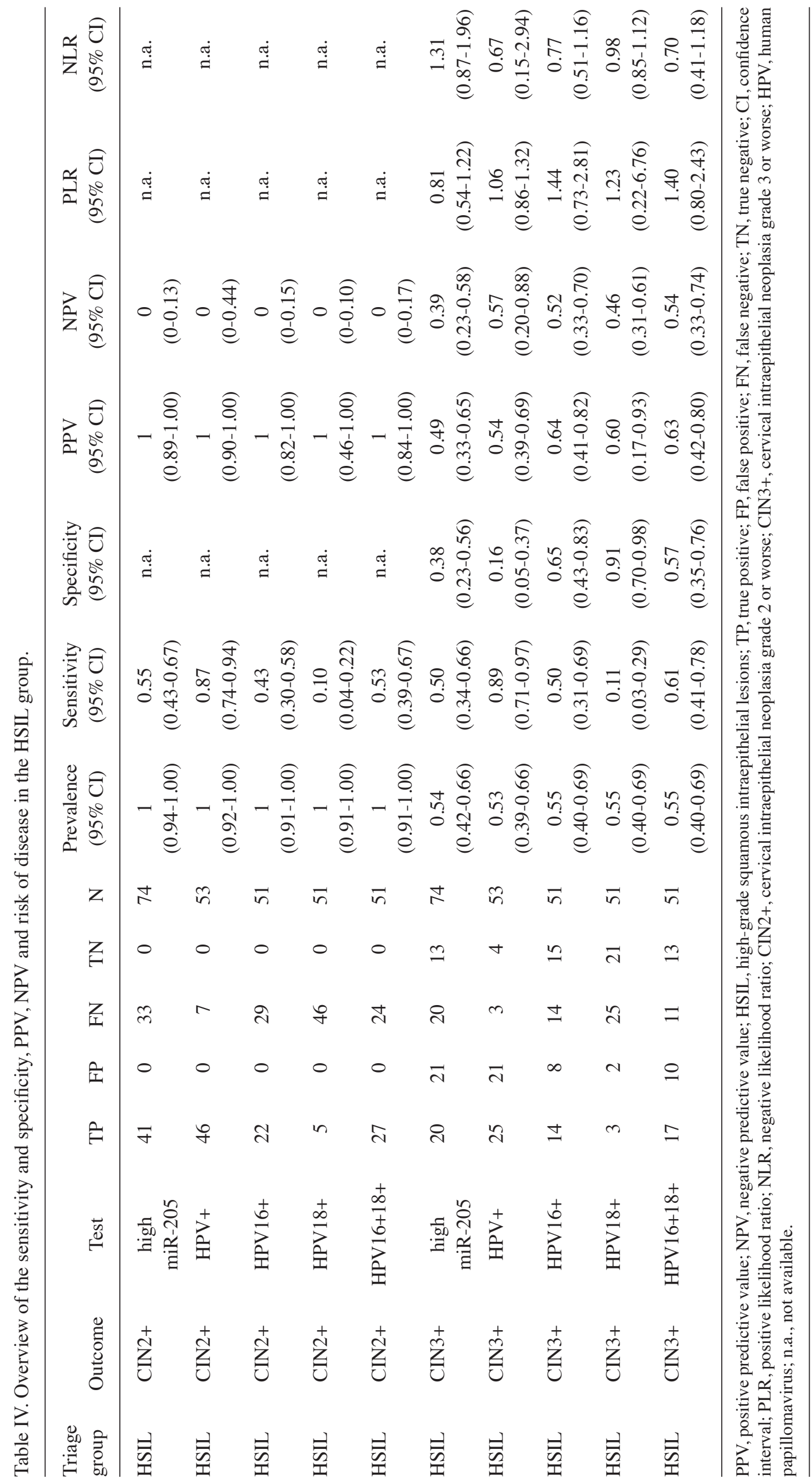


Table V. Correlation of clinical features of LBC samples with miR-205 expression levels.

\begin{tabular}{|c|c|c|c|c|}
\hline Characteristics & All cases & $\begin{array}{l}\text { High miR-205 } \\
\quad(>\text { median })\end{array}$ & $\begin{array}{l}\text { Low miR-205 } \\
(<\text { median })\end{array}$ & P-value \\
\hline \multicolumn{5}{|l|}{ Age $(n=140)$} \\
\hline$<32.5$ & 70 & 39 & 31 & \multirow[t]{2}{*}{0.1763} \\
\hline$>32.5$ & 70 & 31 & 39 & \\
\hline \multicolumn{5}{|l|}{$\operatorname{HPV}(n=115)$} \\
\hline Positive & 93 & 47 & 46 & \multirow[t]{2}{*}{0.7352} \\
\hline Negative & 22 & 12 & 10 & \\
\hline \multicolumn{5}{|l|}{ HPV subtypes (n=90) } \\
\hline HPV16, HPV18 & 43 & 23 & 20 & \multirow[t]{2}{*}{0.5267} \\
\hline Non HPV16, non HPV18 & 47 & 22 & 25 & \\
\hline \multicolumn{5}{|l|}{ Cytology (n=140) } \\
\hline LSIL & 45 & 20 & 25 & \multirow[t]{2}{*}{0.3093} \\
\hline HSIL & 74 & 40 & 34 & \\
\hline \multicolumn{5}{|l|}{ Histology $(\mathrm{n}=123)$} \\
\hline CIN1 & 35 & 17 & 18 & \multirow[t]{2}{*}{0.8391} \\
\hline CIN2+ & 79 & 40 & 39 & \\
\hline \multicolumn{5}{|l|}{ Final diagnosis $(\mathrm{n}=140)$} \\
\hline CIN1 & 29 & 11 & 18 & \multirow[t]{2}{*}{0.1657} \\
\hline CIN2+ & 95 & 50 & 45 & \\
\hline
\end{tabular}

${ }^{a}$ Two-tailed $\chi^{2}$ test (without Yates correlation). High or low miR-205 expression based on the median expression level. LBC, liquid-based cytology; HPV, human papillomavirus; LSIL, low-grade squamous intraepithelial lesion; HSIL, high-grade squamous intraepithelial lesion; CIN1, cervical intra-epithelial neoplasia grade 1; CIN2+, cervical intra-epithelial neoplasia grade 2 or worse.

expression by transcription repression or translation inhibition (38). Dysregulated miRNA profiles have been identified in various human cancer types, including cervical cancer $(17,39)$. However, the majority of previous studies were based on tissue samples or serum samples; there is a lack of knowledge concerning miRNA expression in LBC samples. miR-205 is frequently dysregulated in many cancer types and functions as a either a tumor suppressor or an oncogene, depending on the cellular context (20). miR-205 expression in tumor tissue or serum is associated with the development and progression of tumors (40). Our previous studies revealed that miR-205 is highly expressed in cervical tumor tissue compared with matched normal cervical tissue, and further demonstrated that miR-205 has an oncogenic role by promoting cell proliferation and migration in cervical cancer cells $(17,20)$. In the present study, miR-205 was selected as an example to evaluate the possibility of miRNA detection by RT-qPCR in LBC samples and to assess the potential value of miR-205 in clinical applications.

The preliminary results revealed that high miR-205 expression levels had a significantly higher specificity than HPV testing to predict the absence of CIN2+ or CIN3+ in women with LSIL, whereas the corresponding sensitivities were not significantly different. This demonstrates that there may be promising clinical applications for miR-205 expression. HPV testing is not recommended to triage women with LSIL due to its poor specificity, but this may be improved by the addition of the evaluation of miR-205 expression in these patients.
Certain miRNAs have been associated with HPV infection in cervical cancer. For example, miR-218 was specifically underexpressed in HPV16-positive cervical cancer cell lines, cervical lesions and cancer tissues when compared with HPV-negative C33A cells and normal cervical cells (41). Wang et al (42) revealed that HPV16 E6 expression is regulated via the histone acetyltransferase p300 and reported that increases in the expression of miR-16, miR-25, miR-92a and miR-378, and decreased expression of miR-22, miR-27a, miR-29a and miR-100 may be attributed to the HPV oncoproteins E6 and E7. In the present study, the association between high miR-205 expression and the presence of HPV was also analyzed, but no significant differences were observed, indicating that miR-205 expression is not associated with HPV infection.

In addition, no significant association between $m i R-205$ expression and cancer stage was detected based on cytology, histology or final histopathological diagnosis. This may indicate that miR-205 expression levels do not increase at specific stages, but may increase continually during cancer progression. To better address this question, analyses are required to be performed on more than one sample from the same patient, on specially paired samples or on series of samples.

The present study cohort was taken from patients attending the population-based organized cervical cancer screening program in Sweden, and the majority of the samples were pre-malignant. However, the majority of the cells in the samples were normal, and thus it was difficult to distinguish if the 
miR-205 molecules extracted were from abnormal or normal cells. Theoretically, other single-cell-based detection methods, such as in situ hybridization $(43,44)$ or microfluidic flow cytometry $(45,46)$ are practical and ideal methods for LBC.

In conclusion, the findings from this screening-based population study revealed that high miR-205 expression levels in patients with LSIL provided statistically higher specificity than HPV testing to predict the absence of CIN2+ and CIN3+. Therefore, the data suggest that miRNA detection in LBC samples may have a potential application as an adjunct to HPV testing in the triage of women with LSIL. Further studies in larger cohorts or testing for a panel of miRNAs is required before recommendations may be suggested.

\section{Acknowledgements}

The present study was supported by the Swedish Research Council (grant nos. 523-2009-3517 and 521-2010-3518), the Swedish Cancer Foundation (grant no. CAN2011/471), the King Gustaf V Jubilee Fund and the Cancer Research Funds of Radiumhemmet (grant no. 154022). The authors would like to thank Ms. Trudy Perdrix-Thoma from Professional Standards Editing, Inc. (222 Lovejoy Avenue, Waterloo, IA 50,701, USA), for the English language revision.

\section{References}

1. Ferlay J, Shin HR, Bray F, Forman D, Mathers C and Parkin DM Estimates of worldwide burden of cancer in 2008: GLOBOCAN 2008. Int J Cancer 127: 2893-2917, 2010.

2. The National Board of Health and Welfare: Cancer incidence in Sweden 2008. The National Board of Health and Welfare, Stockholm, 2009.

3. National Quality Register for Cervical Screening: Prevention of cervical cancer in Sweden. Annual report 2014 with data to 2013. National Quality Register for Cervical Screening, Stockholm, Swedish 2014

4. Bosch FX and Muñoz N: The viral etiology of cervical cancer. Virus Res 89: 183-190, 2002.

5. Woodman CB, Collins SI and Young LS: The natural history of cervical HPV infection: Unresolved issues. Nat Rev Cancer 7: 11-22, 2007.

6. Münger K: Disruption of oncogene/tumor suppressor networks during human carcinogenesis. Cancer Invest 20: 71-81, 2002.

7. Pett $\mathrm{M}$ and Coleman N: Integration of high-risk human papillomavirus: A key event in cervical carcinogenesis? J Pathol 212 356-367, 2007.

8. Imaoka H, Toiyama Y, Okigami M, Yasuda H, Saigusa S, Ohi M, Tanaka K, Inoue Y, Mohri Y and Kusunoki M: Circulating microRNA-203 predicts metastases, early recurrence and poor prognosis in human gastric cancer. Gastric Cancer 19: 744-753, 2016.

9. Naucler P, Ryd W, Törnberg S, Strand A, Wadell G, Elfgren K, Rådberg T, Strander B, Johansson B, Forslund O, et al: Human papillomavirus and Papanicolaou tests to screen for cervical cancer. N Engl J Med 357: 1589-1597, 2007.

10. Giorgi-Rossi P, Franceschi S and Ronco G: HPV prevalence and accuracy of HPV testing to detect high-grade cervical intraepithelial neoplasia. Int J Cancer 130: 1387-1394, 2012.

11. Bergström R, Sparén P and Adami HO. Trends in cancer of the cervix uteri in Sweden following cytological screening. Br J Cancer 81:159-166, 1999.

12. Cuzick J, Clavel C, Petry KU, Meijer CJ, Hoyer H, Ratnam S, Szarewski A, Birembaut P, Kulasingam S, Sasieni P and Iftner T: Overview of the European and North American studies on HPV testing in primary cervical cancer screening. Int J Cancer 119 1095-1101, 2006.

13. Nanda K, McCrory DC, Myers ER, Bastian LA, Hasselblad V, Hickey JD and Matchar DB: Accuracy of the Papanicolaou test in screening for and follow-up of cervical cytologic abnormalities: A systematic review. Ann Intern Med 132: 810-819, 2000.
14. Strander B, Andersson-Ellström A, Milsom I, Rådberg T and Ryd W: Liquid-based cytology versus conventional Papanicolaou smear in an organized screening program: A prospective randomized study. Cancer 111: 285-291, 2007.

15. Persson M, Elfström KM, Brismar Wendel S, Weiderpass E and Andersson S: Triage of HR-HPV positive women with minor cytological abnormalities: A comparison of mRNA testing, HPV DNA testing and repeat cytology using a 4-year follow-up of a population-based study. PLoS One 9: e90023, 2014.

16. Bartel DP: MicroRNAs: Genomics, biogenesis, mechanism, and function. Cell 116: 281-297, 2004.

17. Lui WO, Pourmand N, Patterson BK and Fire A: Patterns of known and novel small RNAs in human cervical cancer. Cancer Res 67: 6031-6043, 2007.

18. Wang X, Tang S, Le SY, Lu R, Rader JS, Meyers C and Zheng ZM: Aberrant expression of oncogenic and tumor-suppressive microRNAs in cervical cancer is required for cancer cell growth. PLoS One 3: e2557, 2008.

19. Witten D, Tibshirani R, Gu SG, Fire A and Lui WO: Ultra-high throughput sequencing-based small RNA discovery and discrete statistical biomarker analysis in a collection of cervical tumours and matched controls. BMC Biol 8: 58, 2010.

20. Xie H, Zhao Y, Caramuta S, Larsson C and Lui WO: MiR-205 expression promotes cell proliferation and migration of human cervical cancer cells. PLoS One 7: e46990, 2012.

21. Hagrass HA, Sharaf S, Pasha HF, Tantawy EA, Mohamed RH and Kassem R: Circulating microRNAs-a new horizon in molecular diagnosis of breast cancer. Genes Cancer 6: 281-287, 2015.

22. He Y, Lin J, Kong D, Huang M, Xu C, Kim TK, Etheridge A, Luo Y, Ding $\mathrm{Y}$ and Wang K: Current state of circulating microRNAs as cancer biomarkers. Clin Chem 61: 1138-1155, 2015.

23. Lin XJ, Chong Y, Guo ZW, Xie C, Yang XJ, Zhang Q, Li SP, Xiong Y, Yuan Y, Min J, et al: A serum microRNA classifier for early detection of hepatocellular carcinoma: A multicentre, retrospective, longitudinal biomarker identification study with a nested case-control study. Lancet Oncol 16: 804-815, 2015.

24. Mu YP, Sun WJ, Lu CW and Su XL: MicroRNAs may serve as emerging molecular biomarkers for diagnosis and prognostic assessment or as targets for therapy in gastric cancer. Asian Pac J Cancer Prev 16: 4813-4820, 2015.

25. Nonaka R, Miyake Y, Hata T, Kagawa Y, Kato T, Osawa H, Nishimura J, Ikenaga M, Murata K, Uemura M, et al: Circulating miR-103 and miR-720 as novel serum biomarkers for patients with colorectal cancer. Int J Oncol 47: 1097-1102, 2015.

26. Yu J, Jin L, Jiang L, Gao L, Zhou J, Hu Y, Li W, Zhi Q and Zhu X: Serum miR-372 is a diagnostic and prognostic biomarker in patients with early colorectal cancer. Anticancer Agents Med Chem 16: 424-431, 2016.

27. Orang AV, Safaralizadeh R, Hosseinpour Feizi MA and Somi MH: Diagnostic and prognostic value of miR-205 in colorectal cancer. Asian Pac J Cancer Prev 15: 4033-4037, 2014.

28. Zheng H, Zhang L, Zhao Y, Yang D, Song F, Wen Y, Hao Q, $\mathrm{Hu} \mathrm{Z}$, Zhang $\mathrm{W}$ and Chen $\mathrm{K}$ : Plasma miRNAs as diagnostic and prognostic biomarkers for ovarian cancer. PLoS One 8: e77853, 2013.

29. Yue X, Lan F, Hu M, Pan Q, Wang Q and Wang J: Downregulation of serum microRNA-205 as a potential diagnostic and prognostic biomarker for human glioma. J Neurosurg 124: 122-128, 2016.

30. Ma Q, Wan G, Wang S, Yang W, Zhang J and Yao X: Serum microRNA-205 as a novel biomarker for cervical cancer patients. Cancer Cell Int 14: 81, 2014.

31. Zhao S, Yao D, Chen J and Ding N: Circulating miRNA-20a and miRNA-203 for screening lymph node metastasis in early stage cervical cancer. Genet Test Mol Biomarkers 17: 631-636, 2013.

32. Malta M, Ribeiro J, Monteiro P, Loureiro J, Medeiros R and Sousa H: Let-7c is a candidate biomarker for cervical intraepithelial lesions: A pilot study. Mol Diagn Ther 19: 191-196, 2015.

33. Tian Q, Li Y, Wang F, Li Y, Xu J, Shen Y, Ye F, Wang X, Cheng X, Chen Y, et al: MicroRNA detection in cervical exfoliated cells as a triage for human papillomavirus-positive women. J Natl Cancer Inst 106: pii: dju241, 2014.

34. Fröberg M, Johansson B, Hjerpe A and Andersson S: Human papillomavirus 'reflex' testing as a screening method in cases of minor cytological abnormalities. Br J Cancer 99: 563-568, 2008.

35. Solomon D, Davey D, Kurman R, Moriarty A, O'Connor D, Prey M, Raab S, Sherman M, Wilbur D, Wright T Jr, et al: The 2001 bethesda system: Terminology for reporting results of cervical cytology. JAMA 287: 2114-2119, 2002. 
36. Livak KJ and Schmittgen TD: Analysis of relative gene expression data using real-time quantitative PCR and the 2(-Delta Delta C(T)) method. Methods 25: 402-408, 2001.

37. Canavan TP and Doshi NR: Cervical cancer. Am Fam Physician 61: 1369-1376, 2000.

38. Esquela-Kerscher A and Slack FJ: Oncomirs-microRNAs with a role in cancer. Nat Rev Cancer 6: 259-269, 2006.

39. Lee YS and Dutta A: MicroRNAs in cancer. Annu Rev Pathol 4 199-227, 2009.

40. Qin AY, Zhang XW, Liu L, Yu JP, Li H, Wang SZ, Ren XB and Cao S: MiR-205 in cancer: An angel or a devil? Eur J Cell Biol 92: 54-60, 2013.

41. Martinez I, Gardiner AS, Board KF, Monzon FA, Edwards RP and Khan SA: Human papillomavirus type 16 reduces the expression of microRNA-218 in cervical carcinoma cells. Oncogene 27: 2575-2582, 2008.
42. Wang X, Wang HK, Li Y, Hafner M, Banerjee NS, Tang S, Briskin D, Meyers C, Chow LT, Xie X, et al: MicroRNAs are biomarkers of oncogenic human papillomavirus infections. Proc Natl Acad Sci USA 111: 4262-4267, 2014.

43. Nielsen BS: MicroRNA in situ hybridization. Methods Mol Biol 822: 67-84, 2012

44. Ge J, Zhang LL, Liu SJ, Yu RQ and Chu X: A highly sensitive target-primed rolling circle amplification (TPRCA) method for fluorescent in situ hybridization detection of microRNA in tumor cells. Anal Chem 86: 1808-1815, 2014.

45. Wu M, Piccini M, Koh CY, Lam KS and Singh AK: Single cell microRNA analysis using microfluidic flow cytometry. PLoS One 8: e55044, 2013.

46. Wu M,Piccini ME and Singh AK: MiRNA detection at single-cell resolution using microfluidic LNA flow-FISH. Methods Mol Biol 1211: 245-260, 2014. 